medgen $2016 \cdot 28: 276$

DOI 10.1007/s11825-016-0091-5

(c) Springer-Verlag

Berlin Heidelberg 2016
Österreichische Gesellschaft für Humangenetik
Verantwortlich für den Textteil

Univ.-Prof. Dr. med. Michael Speicher, V.i.S.d.P. Vorsitzender der Österreichischen

Gesellschaft für Humangenetik Institut für Humangenetik der Medizinischen Universität Graz

Harrachgasse 21/8

8010 Graz, Österreich

\title{
Einladung zur 16. Jahrestagung der Österreichischen Gesellschaft für Humangenetik
}

\section{Datum: Freitag, den 30. September 2016 \\ Ort: Institut für Medizinische Genetik, Hauptvorlesungsraum, Währinger Strasse 10, 1090 Wien}

Im Namen der Österreichischen Gesellschaft für Humangenetik

laden wir Sie sehr herzlich zur ÖGH-Jahrestagung 2016 in Wien ein.

\section{Hauptvorträge}

\section{Neugeborenen-Screening}

Biomarker bei neurometabolischen Erkrankungen

Mukopolysaccharidosen im höheren Lebensalter

Primäre Ziliäre Dyskinesie - Genotyp-Phänotyp-Assoziation

Medikamentöse Therapieansätze beim Marfan-Syndrom korreliert das Outcome mit dem Genotyp?

\section{Turner Syndrom}

Phosphatdiabetes

Präimplantationsdiagnostik, Übersicht und Perspektive

Polkörperdiagnostik

Neue Möglichkeiten bei der PID

Praktische Aspekte, Indikationsstellung, Hürden und Besonderheiten aus der Praxis
Johannes Zschocke

Barbara Plecko

Susanne Kircher

Susanne Diesner

Christiane Pees

Gabriele Häusler

Albert Raimann

Ute Hehr

Jürgen Neesen

Wilfried Feichtinger

Andreas Obruca

Wir freuen uns über Ihre Beiträge (bitte an Brigitte.Hofer@meduniwien.ac.at)

und viele interessierte Teilnehmer (Details zur Tagung folgen per Rundmail an alle ÖGH- Mitglieder).

Beste Grüße aus Wien,

Franco Laccone 